\title{
Insulin secretion-sensitivity index-2 could be a novel marker in the identification of the role of pancreatic iron deposition on beta-cell function in thalassemia major
}

\author{
Nihal Karadas ${ }^{1)}$, Banu Yurekli2), Selen Bayraktaroglu ${ }^{3)}$ and Yesim Aydinok ${ }^{1)}$ \\ 1) Department of Pediatric Hematology \& Oncology, Ege University Faculty of Medicine, Izmir, Turkey \\ 2) Department of Endocrinology, Ege University Faculty of Medicine, Izmir, Turkey \\ 3) Department of Radiology, Ege University Faculty of Medicine, Izmir, Turkey
}

\begin{abstract}
The purpose of this study is to evaluate the impact of insulin secretion-sensitivity index-2 (ISSI-2) in the identification of the role of pancreatic iron deposition on beta-cell function in thalassemia major. Tissue iron stores were measured with magnetic resonance imaging (MRI) in the liver (R2), pancreas (R2*), and heart (T2*). ISSI-2 was assessed as a novel oral glucose tolerance test-based measure of beta-cell function. Also, the Stumvoll index showing the insulin sensitivity and Stumvoll index estimating first and second phase insulin secretion were calculated. Fourteen of the 51 Thalassemia Major patients, aged 8-34 (mean $21.1 \pm 7.2)$ years-old, had either an impaired glucose tolerance test $(n=9,17.6 \%)$ or diabetes mellitus $(n=5,9.8 \%)$ - referred to as the glucose dysregulation (GD) group. The median serum ferritin and the mean liver R2 and cardiac T2* values were not significantly different between the GD and normal glucose tolerance (NGT, $n=37$ ) groups whereas pancreas R2* was significantly higher in the GD group compared to the NGT group $(p=0.004)$. Patients with GD showed significantly lower ISSI-2 index $(p<0.001)$ as well as the Stumvoll index and Stumvoll first and second phase indices compared to those with NGT $(p<0.001)$. All patients with GD displayed a pancreas R2 $>50 \mathrm{~Hz}$ and ISSI-2 $<2$. In conclusion, Pancreas R2* MRI combined with ISSI-2 index may be valuable parameters to identify patients at the highest risk for developing glucose dysregulation.
\end{abstract}

Key words: Thalassemia, Glucose dysregulation, Pancreatic iron, Beta cell function

REGULARLY transfused patients with Thalassemia Major (TM) may develop iron overload early in their transfusion history unless it is controlled by a proper iron chelation therapy. Susceptibility to iron toxicity differs between organs, with the most vulnerable being the endocrine glands, the heart, and the liver [1-4]. Non-

Submitted Jun. 1, 2019; Accepted Jul. 30, 2019 as EJ19-0191 Released online in J-STAGE as advance publication Sep. 13, 2019 Correspondence to: Yesim Aydinok, Department of Pediatric Hematology \& Oncology, Ege University Faculty of Medicine, Kazimdirik Mahallesi, Universite Cd. No:9, 35100, Bornova, Izmir, Turkey.

E-mail: yesim.aydinok@yahoo.com

Appendix: ISSI-2, insulin secretion-sensitivity index-2; SF, serum ferritin; MRI, magnetic resonance imaging; CMR, Cardiovascular Imaging Solutions; GD, glucose dysregulation; LIC, liver iron concentration; NGT, normal glucose tolerance; $\mathrm{HCV}$, hepatitis $\mathrm{C}$ virus; TM, Thalassemia Major; BMI, body mass index; DM, diabetes mellitus; IDDM, insulin-dependent diabetes mellitus; OGTT, oral glucose tolerance test; RBCC, red blood cell concentrate; IFG, impaired fasting glucose; DFX, deferasirox; IGT, impaired glucose tolerance; FSIVGTT, frequently sampled intravenous glucose tolerance test; AUC, area under the curve; IGI, insulinogenic index; HOMA-IR, Homeostatic Model Assessment of Insulin Resistance; HOMA-B, Homeostatic Model Assessment of $\beta$-cell function invasive estimation of myocardial siderosis by magnetic resonance imaging (MRI) and appropriate intensification of iron chelation therapy prior to the onset of cardiac dysfunction have improved survival [5-7], but endocrinopathies still account for significant morbidity in TM [8-13]. Of the endocrinopathies, diabetes mellitus (DM) is one of those leading to significant morbidity, reduced quality of life, and life expectancy. Prevalence has been reported between 2.3 and $24 \%$ [8-15].

It is suggested that iron-mediated pancreatic $\beta$-cell damage leading to insulin deficiency and preceding insulin resistance due to liver fibrosis contribute to glucose dysregulation (GD) in TM $[15,16]$. Early biochemical detection of abnormal glucose metabolism is important to prevent overt diabetes mellitus by appropriate clinical management [17]. Pancreatic MRI studies demonstrated that severe pancreatic iron deposition may occur as early as the first decade of life. It is assumed that there would be a therapeutic window between clinically silent iron deposition and irreversible pancreatic damage during which an appropriate intensification of iron chelation may save the organ function [18]. Although, demonstra- 
tion of prospective risk associated with pancreatic iron deposition and organ function will be required longitudinal studies, a proper biochemical assessment of beta-cell function may be useful to integrate pancreas R2* values with the tissue damage. In this study, we aimed to explore the value of insulin secretion-sensitivity index-2 (ISSI-2) as a novel oral glucose tolerance test (OGTT)based measure of beta-cell function analogous to the disposition index and its relationship with pancreatic iron deposition.

\section{Patients and Methods}

The study was conducted at a specialised thalassemia clinical centre with Good Clinical Practice compliance and in accordance with the Helsinki Declaration on human subject research. The study was approved by the Ege University Ethics Committee. The inclusion criteria were the diagnosis of TM with management in a chronic transfusion program for at least eight transfusions per year, zeight years, and signed informed consent or paediatric assent as required. The medical history of patients with respect to transfusion and chelation, transfusiontransmitted infections, spleen status was recorded, liver function tests and lipid profiles were assessed.

All patients participated in a frequently sampled OGTT. The blood samples were drawn after at least 12 hours of fasting overnight at $-15 \mathrm{~min}$ and $0 \mathrm{~min}$ for glucose, insulin, and C-peptide levels to provide the stability of the baseline glycaemic state. Following the baseline sampling, $1.75 \mathrm{~g} / \mathrm{kg}$ (max: $75 \mathrm{~g}$ ) of glucose solution was given to the patients. Glucose, insulin, and C-peptides were measured at 30,45,60,90, 120, 150, and 180 minutes after an oral glucose load. Patients were classified as having normal glucose tolerance (NGT), impaired fasting glucose (IFG), impaired glucose tolerance (IGT) or DM, according to the American Diabetes Association criteria [19]. From the results of the OGTT, Matsuda and Stumvoll indices and ISSI-2 were calculated by using published formulas as indices of insulin sensitivity and secretion.

The Matsuda index represents whole-body insulin sensitivity. It was calculated by using the web calculator [20]. We calculated the area under the curve (AUC) using the trapezoidal rule for glucose, insulin, and Cpeptide at $0,30,60,90,120,150$, and $180 \mathrm{~min}$ [21]. The Stumvoll index showed insulin sensitivity, and the Stumvoll first phase and second phase insulin secretion were calculated as indicated by Stumvoll et al. (Stumvoll index $0.149-0.000467 \times$ ins0 $-0.00466 \times$ gluc60 , Stumvoll 1 st phase $1,283+1.829 \times$ ins $30-138.7 \times$ gluc30 $+3.772 \times$ ins0, Stumvoll 2 nd phase $287+0.4164$ $\times$ ins $30-26.07 \times$ gluc30 $+0.9226 \times$ ins0) [22]. ISSI-2 was calculated according to the formula: (AUC insulin/AUC glucose) $\times$ Matsuda Index [23]

The average yearly serum ferritin (SF) levels of subjects were calculated. Myocardial T2* was assessed using a cardiac gated single breath-hold, multi-echo gradient echo sequence as previously described [24]. In brief, a single $10 \mathrm{~mm}$ midventricular short axis slice was acquired at 8 echo times $(2.6-22.3 \mathrm{~ms})$ in a single breathhold. A full-thickness region of interest was chosen in the LV septum for analysis as previously detailed [25]. The signal intensity of this region at each echo time was measured with the European Union-approved software CMR (Cardiovascular Imaging Solutions) tools. Cardiac $\mathrm{T} 2 *<20 \mathrm{~ms}$ and corresponding $\mathrm{R} 2 *>50 \mathrm{~Hz}$ were used to represent significant cardiac iron [25]. Liver R2 and Pancreas R2* were measured and analysed as described previously $[18,26]$. Liver R2 values were converted to predict liver iron concentration (LIC) using validated calibration curves [26]. Pancreas R2* of $30 \mathrm{~Hz}$ was accepted as the upper limit of normal pancreas iron, and $>100 \mathrm{~Hz}$ was the cutoff which has previously been associated with an increased risk of cardiac iron loading [18]. Cardiac R2*, equal to 1,000/T2*, was used for statistical comparison of cardiac iron with pancreatic iron. MRI sequences of patients were obtained by the same radiology technician and analysed by the same radiologist.

\section{Statistical analysis}

Results are expressed as the mean \pm standard deviation unless otherwise stated. The independent-samples $t$-test or Mann-Whitney $U$ test were performed wherever appropriate to search for differences between the two groups according to the normality tests. Pearson or Spearman's correlation was performed based on the pattern of distribution to examine how variables were related. Logistic regression was performed to measure the relationship between the categorical dependent variable and one or more independent variables. For those statistical analyses, SPSS 21.0 (Statistical Package for the Social Sciences) was used and $p<0.05$ was accepted as statistically significant.

\section{Results}

Fifty-one patients (21 male and 30 female) aged between 8-34 years were recruited to the study. A total of 19 out of 51 patients (37\%) had undergone splenectomy. Two patients had a medical history of chronic hepatitis $\mathrm{C}$ virus (HCV) infection, but $\mathrm{HCV}$ clearance had been achieved in both by antiviral therapy, and HCVRNA remained negative over 10 years. Alanine aminotransferase levels were slightly increased in three patients but not above two times of the upper limit of 
Table 1 Demographics of the total study population and those with normal glucose tolerance and glucose dysregulations

\begin{tabular}{|c|c|c|c|c|}
\hline Variables & Total & NGT & GD & $p$ value* \\
\hline Sample size $(n)$ & 51 & 37 & 14 & \\
\hline Age (years) & $21.1 \pm 7.2(8-34)$ & $19.0 \pm 6.56(8-34)$ & $26.7 \pm 5.57(16.0-33.5)$ & 0.000 \\
\hline $\operatorname{BMI}\left(\mathrm{kg} / \mathrm{m}^{2}\right)$ & $20.4 \pm 3.7(18.5-33.3)$ & $19.5 \pm 3.2(14.0-28.5)$ & $22.8 \pm 3.8(17.9-33.3)$ & 0.004 \\
\hline Ferritin (ug/dL) & $2,260 \pm 2,601(183-13,301)$ & $2,245 \pm 2,731(291-13,300)$ & $2,301 \pm 2,316(183-8,685)$ & 0.946 \\
\hline Pancreas R2* (Hz) & $73.0 \pm 43.6(18.8-170.4)$ & $62.8 \pm 40.8(18.8-155.9)$ & $104.2 \pm 37.6(59.7-170.4)$ & 0.004 \\
\hline$<30 \mathrm{~Hz}(\%)$ & 20.0 & 27.3 & none & \\
\hline$\geq 30-100 \mathrm{~Hz}(\%)$ & 49.0 & 48.5 & 53.8 & \\
\hline >100 Hz (\%) & 31.0 & 24.2 & 46.2 & \\
\hline Cardiac T2* (msec) & $23.4 \pm 7.6(4.2-42.5)$ & $23.9 \pm 6.2(6.2-38.0)$ & $22.1 \pm 10.7(4.2-42.5)$ & 0.478 \\
\hline LIC (mg/g d.w.) & $8.8 \pm 11.9(0.4-53.2)$ & $7.7 \pm 9.7(0.78-43.7)$ & $11.5 \pm 16.0(0.4-53.2)$ & 0.318 \\
\hline
\end{tabular}

NGT, Normal glucose tolerance; GD, Glucose dysregulation; BMI, Body mass index.

Continuous variables were expressed as mean \pm standard deviation (range) but $\mathrm{SF}$ as median.

*the mean difference between NGT and GD by paired $t$-test.

Table 2 Iron loading status of the study population

\begin{tabular}{lccc}
\hline Iron overload & optimum & moderate & severe \\
\hline Ferritin (ug/dL) [27, 28] & $<1,000$ & $\geq 1,000-2,500$ & $>2,500$ \\
$\%$ & 47 & 25.5 & 27.5 \\
LIC (mg/g d.w.) [29, 30] & $<7$ & $\geq 7-15$ & $>15$ \\
$\%$ & 60 & 22 & 18 \\
Cardiac T2* (msec) [25] & $>20$ & $\geq 10-20$ & $<10$ \\
$\%$ & 70.6 & 23.5 & 5.9 \\
Pancreas R2* (Hz) [18] & $<30$ & $\geq 30-100$ & 100 \\
$\%$ & 20 & 49 & 31 \\
\hline
\end{tabular}

normal and none of the patients presented with cholestasis. Hypertriglyceridemia was observed in eight (15.6\%) patients, and none of them had hypercholesterolemia. Demographics of the overall study population and those with NGT and GD are presented in Table 1. The body mass index (BMI) of the cohort was within the normal range in all but three; the patient with insulin-dependent DM (IDDM) was obese (BMI; $33.3 \mathrm{~kg} / \mathrm{m}^{2}$ ) and the other with IGT and NGT were overweight (BMI; 27.1 and $28.5 \mathrm{~kg} / \mathrm{m}^{2}$, respectively) as classified according to the World Health Organization Criteria. At baseline, all patients were receiving regular red blood cell concentrate (RBCC) transfusions for $17.7 \pm 7.34$ (median 18.25) years and concomitant iron chelation therapy. The overall, $95 \%$ of the cohort was on deferasirox (DFX) monotherapy with the average daily dose of $30 \pm 7.34$ (range 13-40) $\mathrm{mg} / \mathrm{kg}$ body weight. The distribution of median SF levels and tissue iron loading assessed by MRI was broad. The overall frequency of subjects with optimum, moderate, and severe SF and organ-specific iron distribution is shown in Table 2. Despite that tissue-specific MRI studies demonstrated optimum liver $(60 \%)$ and cardiac (70.6\%) iron loading in the majority of cohorts, the pancreatic iron deposition was moderate to severe in $80 \%$ of the patients based on pre-defined criteria.

Nine of 51 patients had IGT (17.6\%) and 5 patients (9.8\%) had DM. No patients demonstrated isolated IFG. A total of $14(27.4 \%)$ subjects had GD including IGT and DM whereas 37 subjects NGT. Two patients with DM had a history of IGT who were receiving oral hypoglycaemic agent which was omitted one week prior to OGTT, and one was suffering from IDDM in whom OGTT was not applied.

There was no statistically significant difference with respect to mean SF, LIC, and cardiac T2* levels between the cohorts of NGT and GD. However, the patients with GD demonstrated a significantly elevated mean pancreas R2* (35.5-170.4 Hz) compared to those with NGT $(18.8-155.9 \mathrm{~Hz})(p=0.004)$. None of the patients with GD but $27.3 \%$ of patients with NGT had a normal pancreas R2* of $<30 \mathrm{~Hz}$. Nevertheless, the majority (72.7\%) of patients with NGT revealed either moderate $(\geq 30-100$ $\mathrm{Hz})$ or severe $(>100 \mathrm{~Hz})$ pancreatic siderosis. The mean age of patients with GD was significantly higher compared to those with NGT and ranged between 16-33 years vs. 8-34 years in the cohorts with GD and NGT, respectively. Patients with GD had significantly higher BMI compared to those with NGT (Table 1). However, multiple logistic regression analysis demonstrated that pancreas R2* was an independent predictor of glucose tolerance status when age and BMI included $(r 2=0.39$, $p=0.048$; OR, 1.020 (1.000-1.041) 95\% CI).

There was a weak but significant correlation between pancreas R2* and age $(r=0.321, p<0.04)$. The pancreas R2* showed a significant correlation with cardiac R2* $(r$ 
Table 3 Comparison of insulin secretion and sensitivity indexes between NGT and GD group

\begin{tabular}{lccc}
\hline Indexes & NGT & GD & $p$ value \\
\hline FPG $(\mathrm{mg} / \mathrm{dL})$ & $78.7 \pm 10.0(47-96)$ & $92.5 \pm 18.7(70-138)$ & 0.002 \\
Fasting insülin $(\mathrm{mIU} / \mathrm{mL})$ & $6.2 \pm 4.5(2.0-27.1)$ & $6.8 \pm 4.2(2.0-12.4)$ & 0.43 \\
Fasting C-peptide $(\mathrm{ng} / \mathrm{mL})$ & $2.1 \pm 0.7(0.8-3.8)$ & $3.7 \pm 2.9(1.2-16.3)$ & 0.14 \\
\hline AUCins & $164.3 \pm 81.9(32.2-397.4)$ & $172.5(37.1-348.7)$ & 0.66 \\
AUCglu & $649.0 \pm 81.8(464-848)$ & $1,011.4 \pm 250.0(770-1,534)$ & 0.000 \\
AUCins/glu & $0.24 \pm 0.10(0.06-0.48)$ & $0.17 \pm 0.09(0.05-0.42)$ & 0.045 \\
AUC C-peptide & $46.1 \pm 13.5(27.2-82.8)$ & $52.6 \pm 11.6(32.2-75.3)$ & 0.14 \\
Matsuda index & $10.7 \pm 7.2(2.7-37.9)$ & $8.9 \pm 7.6(2.74-30.5)$ & 0.47 \\
ISSI-2 & $2.11 \pm 0.81(1.18-4.81)$ & $1.19 \pm 0.46(0.26-1.70)$ & 0.000 \\
Stumvoll index & $0.14 \pm 0.01(0.11-0.17)$ & $0.11 \pm 0.01(0.08-0.13)$ & 0.000 \\
Stumvoll 1. phase & $1,029.4 \pm 464.1(469.6-3,003.1)$ & $469.2 \pm 333.0(74.2-1,216.0)$ & 0.000 \\
Stumvoll 2. phase & $272.4 \pm 113.6(142.1-773.1)$ & $147.7 \pm 76.6(60.7-321.6)$ & 0.000 \\
\hline
\end{tabular}

NGT, Normal glucose tolerance; GD, Glucose dysregulation; FPG, Fasting plasma glucose; AUC, Area under the curve; ISSI-2, Insulin sensitivity and secretion index. $P<0.05$ was significant.

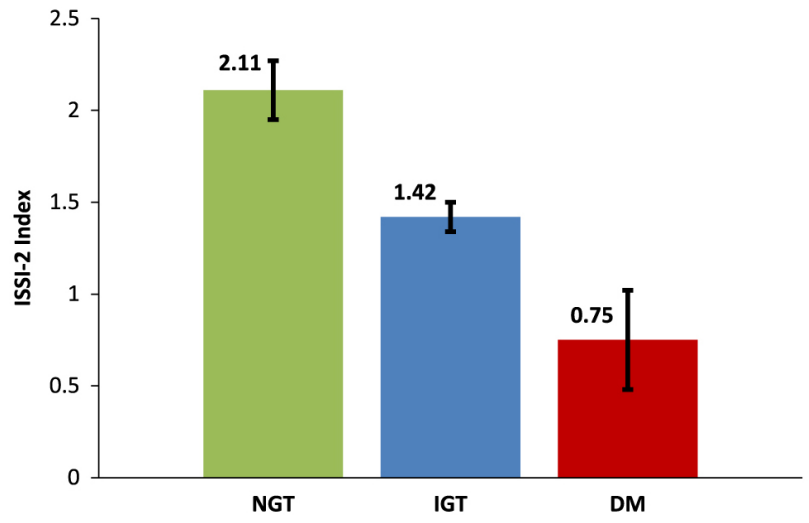

Fig. 1 Distribution of insulin secretion - sensitivity index-2 values in thalassemia major patients.

ISSI-2: insulin secretion - sensitivity index-2; NGT: normal glucose tolerance and represented in green; IGT: impaired glucose tolerance represented in blue; DM: diabetes mellitus represented in red.

$=0.369, p=0.014)$. However, no significant correlation was observed between pancreas R2* and LIC ( $r=0.087$, $p=0.57)$ or SF $(r=0.238, p=0.11)$. Although, in general, cardiac iron deposition depicted by cardiac R2* $>50$ $\mathrm{Hz}(\mathrm{T} 2 *<20 \mathrm{~ms})$ was observed in patients with pancreas $\mathrm{R} 2 *>100 \mathrm{~Hz}$, there were a few exceptions (Supplementary Fig. 1).

Insulin indices showing insulin sensitivity and secretion in NGT and GD groups are presented in Table 3. The Matsuda index indicating insulin sensitivity was not different between the groups but the ISSI-2 index estimating beta-cell function was significantly lower in the GD group $(p<0.001)$ (Fig. 1). It was shown that none of

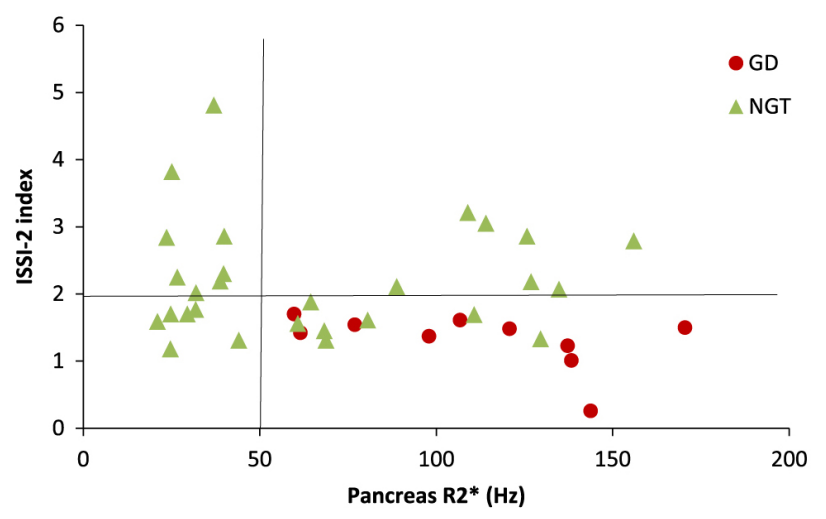

Fig. 2 Distribution of thalassemia patients according to insulin secretion-sensitivity index-2 and pancreatic R2* values.

ISSI-2: Insulin secretion - sensitivity index-2. Patients with normal glucose tolerance (NGT) are shown in green and glucose dysregulation (GD) in red. The patients with GD are those with ISSI- $2 \leq 2$ and pancreatic R2* $>50 \mathrm{~Hz}$.

the patients with ISSI- $2 \geq 2$ or Pancreas R2 $* 50 \mathrm{~Hz}$ had GD. Although, all patients with GD had both ISSI- $2<2$ and pancreas $\mathrm{R} 2 *>50 \mathrm{~Hz}, 25 \%$ of the NGT cohort also displayed the same profile (Fig. 2). The Stumvoll index estimating insulin sensitivity and Stumvoll first phase and second phase insulin secretion indices were significantly lower in the GD group ( $p<0.001, p<0.001$, respectively) (Table 3 ). Insulin sensitivity (Stumvoll index) as a function of insulin release (Stumvoll first phase insulin secretion) demonstrated a progressive decline in insulin sensitivity in patients with IGT and DM who exhibited gradually less insulin secretion (Supplementary Fig. 2). 


\section{Discussion}

In the current relatively young cohort of TM, 14 of 51 patients $(27 \%)$ showed glucose dysregulation. The patients with DM were composed of $9.8 \%$ of the study population and no patient aged 25 years or younger had DM. In contrast, DM was reported as young as 16 years old in larger North American cohorts and 10\% of patients aged between 16-24 years old had DM [8]. The upper limit of age in our cohort resembled the Italian cohort in which the prevalence of complications in TM patients born after 1970 was reported in 2005 and DM was observed in $6 \%$ of patients aged below 35 years [31]. In our cohort, IGT was observed by the age of 16 years old and overall patients with GD were significantly older compared to the patients with NGT. This is consistent with the knowledge that ageing is a strong contributor to developing GD. Obesity is another contributor to the development of GD in adolescents and young adults [32]. Patients with GD had higher BMI compared with the NGT group and family history for diabetes was present in $64 \%$ of patients with GD.

In clinical studies from different geographic regions of the world, high serum ferritin levels and poor compliance with chelation therapy were suggested as the main risk factor associated with endocrine dysfunctions in TM $[11,13,33]$. In our cohort, the mean serum ferritin levels and LIC values were not significantly different between patients with NGT and GD. Au et al. [34] suggested that cardiac $\mathrm{T} 2 *$ was a better predictor of pancreatic dysfunction compared to pancreas $\mathrm{T} 2 *$. In the current study, the cardiac $\mathrm{T} 2 *$ did not have predictive power for identifying $\mathrm{GD}$, but the pancreas $\mathrm{R} 2 *$ value was the strongest predictor of GD. The studies assessing Pancreas R2* or T2* suggested its either a strong or modest linear relationship with cardiac R2* or $\mathrm{T} 2 *[18,34,35]$. In fact, the pancreas R2* did not show a correlation with SF and LIC, but the cardiac R2* was correlated. L-type $\mathrm{Ca}^{2+}$ channels which have an important role in mediating cellular nontransferrin bound iron uptake, are present in large numbers in cardiomyocytes and pancreatic beta cells. Therefore, it is suggested that similar iron loading mechanisms of the pancreas and heart may explain more closely the correlation in the iron burden of these organs [36]. Noetzli et al. suggested that the pancreas appears to increase its iron deposition earlier than the heart and cardiac R2* may not be increased above $50 \mathrm{~Hz}$ (cardiac T2* $<20 \mathrm{~ms}$ ) unless the pancreas R2* exceeds $100 \mathrm{~Hz}$ [18]. Although the majority of patients with increased cardiac iron had significant pancreatic iron loading $(>100 \mathrm{~Hz})$, three out of 11 patients $(27 \%)$ with cardiac R2* slightly above $50 \mathrm{~Hz}(\mathrm{~T} 2 * 19.9,19.4$ and $16.9 \mathrm{~ms})$ had NGT and the corresponding pancreas R2* values were 44,30 , and
$44 \mathrm{~Hz}$, respectively. We would suggest examining cardiac iron deposition by MRI in all patients with evidence of pancreatic iron accumulation depicted by pancreas $\mathrm{R} 2 *>30 \mathrm{~Hz}$.

It has been shown in a previous study that none of the patients with pancreas $\mathrm{R} 2 *<100 \mathrm{~Hz}$ revealed any presentation of GD [37]; however, GD was observed in patients with pancreas R2* as low as $60 \mathrm{~Hz}$ in the current cohort.

Pancreatic beta-cells compensate for the changes in insulin sensitivity by a proportionate and reciprocal change in insulin secretion [38, 39]. So, Bergman and colleagues [40] defined beta cell function between insulin secretion and insulin sensitivity as a hyperbolic function which was confirmed in humans by Kahn et al. [41] stating that insulin secretion must be evaluated in association with insulin sensitivity.

In the study of Retnakaran et al. [42], it was indicated that the actual disposition index provided useful information about the beta-cell function. But its calculation required frequently sampled intravenous glucose tolerance test (FSIVGTT). Two OGTT-based measurements of beta-cell function analogous to actual disposition index have been presented recently. One of them is ISSI-2 and the other one is the disposition index indicated as the insulinogenic index (IGI). In the study of Retnakaran et al. [42], two indices were compared with the actual disposition index derived from FSIVGTT. It was shown that ISSI-2 exhibited stronger correlations with the disposition index than did IGI. Sometimes, IGI could not be calculated accurately because of the paradoxical decrease in insulin levels during the first $30 \mathrm{~min}$ OGTT which is a possible confounding parameter for the use of IGI in the assessment of beta-cell function [42]. Based on the hyperbolic relationship between insulin secretion and insulin sensitivity; ISSI-2 determines the degree of glucose tolerance providing the beta-cell function. Through progression from NGT to IGT and diabetes, ISSI-2 decreases pointing to the deterioration of beta cell compensation for existing insulin resistance. ISSI-2, which could be calculated easily from OGTT was derived from the product of the AUC insulin/glucose pointing to insulin secretion and the Matsuda index which was more helpful than HOMA-IR (homeostatic model assessment-insulin resistance) as it provided whole-body insulin sensitivity [22]. So, we showed for the first time that ISSI-2, indicating beta-cell function was significantly lower in the GD group compared to the NGT in thalassemia patients. Further, all TM patients presenting with GD had a pancreas R2* value greater than $50 \mathrm{~Hz}$ combined with ISSI-2 less than 2. The considerable number of patients with NGT but with a pancreas $\mathrm{R} 2 *$ value of $>50 \mathrm{~Hz}$ and ISSI- $2<2$ may indicate 
those with the highest risk of developing GD. On the other hand, the patients with ISSI- $2>2$ but corresponding pancreas R2* values indicating significant iron deposition may indicate those with silent pancreatic iron deposition with preserved beta-cell function (Supplementary Fig. 1). The Stumvoll index showing insulin sensitivity and Stumvoll first and second phase indices estimating insulin secretion were significantly lower in the GD group compared with the NGT group. We depicted that the Stumvoll first phase insulin secretion was lower at the lowest level of the Stumvoll index representing hyperbolic function (Supplementary Fig. 2).

In the literature, it has been observed that Homeostatic Model Assessment of Insulin Resistance (HOMA-IR) and $\beta$-cell function (HOMA-B) were used mostly in thalassemia patients. Unfortunately, those indices are not enough to evaluate beta-cell function in thalassemia, because HOMA-IR and HOMA-B are only based on fasting glucose and insulin levels. HOMA cannot evaluate the incretin effect which points to glucose-dependent insulin secretion. There was only one study in the literature using the disposition index derived from the Stumvoll first phase insulin secretion and insulin sensitivity calculated from a minimal model approach in thalassemia patients [22]. ISSI-2 could be a good index to evaluate beta-cell function showing a hyperbolic rela- tionship between insulin sensitivity and secretion in thalassemia patients. Besides, the potential of being calculated easily with OGTT is another advantage of ISSI-2 compared to indices calculated from minimal models.

The limitations of our study include the lack of separate DM and IGT groups due to the small number of patients in each group and the cross-sectional nature of the study.

In conclusion, the pancreatic R2* value was a predictor of glucose tolerance status. ISSI-2 was significantly lower in the glucose dysregulation group showing impaired beta-cell function. A pancreas R2* value $>50$ $\mathrm{Hz}$ together with an ISSI- $2<2$ could be valuable parameters to identify patients at risk of developing glucose dysregulation associated with those parameters. Longitudinal studies are needed to disclose the significance of these parameters as early identifiers of patients at risk of developing GD and whether the progression to DM would be reversed.

\section{Acknowledgement}

None of authors has conflict of interest. This research did not receive any specific grant from funding agencies in the public, commercial, or not-for-profit sectors.

\section{References}

1. Berdoukas V, Nord A, Carson S, Puliyel M, Hofstra T, et al. (2013) Tissue iron evaluation in chronically transfused children shows significant levels of iron loading at a very young age. Am J Hematol 88: E283-E285.

2. Wood JC, Origa R, Agus A, Matta G, Coates TD, et al. (2008) Onset of cardiac iron loading in pediatric patients with thalassemia major. Haematologica 93: 917-920.

3. Noetzli LJ, Panigrahy A, Mittelman SD, Hyderi A, Dongelyan A, et al. (2012) Pituitary iron and volume predict hypogonadism in transfusional iron overload. $\mathrm{Am} \mathrm{J}$ Hematol 87: 167-171.

4. Yang G, Liu R, Peng P, Long L, Zhang X, et al. (2014) How early can myocardial iron overload occur in beta thalassemia major? PLoS One 9: e85379.

5. Modell B, Khan M, Darlison M, Westwood MA, Ingram $\mathrm{D}$, et al. (2008) Improved survival of thalassaemia major in the UK and relation to $\mathrm{T} 2 *$ cardiovascular magnetic resonance. J Cardiovasc Magn Reson 10: 42.

6. Telfer P, Coen PG, Christou S, Hadjigavriel M, Kolnakou A, et al. (2006) Survival of medically treated thalassemia patients in Cyprus. Trends and risk factors over the period 1980-2004. Haematologica 91: 1187-1192.

7. Borgna-Pignatti C, Cappellini MD, De Stefano P, Del Vecchio GC, Forni GL, et al. (2006) Cardiac morbidity and mortality in deferoxamine or deferiprone-treated patients with thalassemia major. Blood 107: 3733-3737.
8. Cunningham MJ, Macklin EA, Neufeld EJ, Cohen AR, Thalassemia Clinical Research Network (2004) Complications of beta-thalassemia major in North America. Blood 104: 34-39.

9. De Sanctis V, Elsedfy H, Soliman AT, Elhakim IZ, Soliman NA, et al. (2016) Endocrine profile of $\beta$ thalassemia major patients followed from childhood to advanced adulthood in a tertiary care center. Indian $J$ Endocrinol Metab 20: 451-459.

10. Fung EB, Harmatz PR, Lee PD, Milet M, Bellevue R, et al. (2006) Increased prevalence of iron-overload associated endocrinopathy in thalassaemia versus sickle-cell disease. Br J Haematol 135: 574-582.

11. Toumba M, Sergis A, Kanaris C, Skordis N (2007) Endocrine complications in patients with Thalassaemia Major. Pediatr Endocrinol Rev 5: 642-648.

12. Vichinsky E, Butensky E, Fung E, Hudes M, Theil E, et al. (2005) Comparison of organ dysfunction in transfused patients with SCD or beta thalassemia. Am J Hematol 80: $70-74$.

13. Gamberini MR, De Sanctis V, Gilli G (2008) Hypogonadism, diabetes mellitus, hypothyroidism, hypoparathyroidism: incidence and prevalence related to iron overload and chelation therapy in patients with thalassaemia major followed from 1980 to 2007 in the Ferrara Centre. Pediatr Endocrinol Rev 6 Suppl 1: 158-169. 
14. Li MJ, Peng SS, Lu MY, Chang HH, Yang YL, et al. (2014) Diabetes mellitus patients with thalassemia major. Pediatr Blood Cancer 61: 20-24.

15. Cario H, Holl RW, Debatin KM, Kohne E (2003) Insulin sensitivity and beta-cell secretion in thalassemia major with secondary haemochromatosis: assessment by oral glucose tolerance test. Eur J Pediatr 162: 139-146.

16. Angelopoulos NG, Zervas A, Livada S, Adamopoulos I, Giannopoulos D, et al. (2006) Reduced insulin secretion in normoglycemic patients with beta-thalassemia major. Diabet Med 23: 1327-1331.

17. Jones TW, Boulware SD, Caprio S, Merkel P, Amiel SA, et al. (1993) Correction of hyperinsulinemia by Glyburide treatment in nondiabetic patients with thalassemia major. Pediatr Res 33: 497-500.

18. Noetzli LJ, Papudesi J, Coates TD, Wood JC (2009) Pancreatic iron loading predicts cardiac iron loading in thalassemia major. Blood 114: 4021-4026.

19. The Expert Committee on the Diagnosis and Classification of Diabetes Mellitus (1997) Report of the Expert Committee on the Diagnosis and Classification of Diabetes Mellitus. Diabetes Care 20: 1183-1197.

20. Matsuda M, De Fronzo RA (1999) Insulin sensitivity indices obtained from oral glucose tolerance testing: comparison with the euglycemic insulin clamp. Diabetes Care 22: 1462-1470.

21. Matthews JN, Altman DG, Campbell MJ, Royston P (1990) Analysis of serial measurements in medical research. BMJ 300: 230-235.

22. Stumvoll M, Mitrakou A, Pimenta W, Jenssen T, YkiJärvinen $\mathrm{H}$, et al. (2000) Use of the oral glucose tolerance test to assess insulin release and insulin sensitivity. Diabetes Care 23: 295-301.

23. Retnakaran R, Shen S, Hanley AJ, Vuksan V, Hamilton $\mathrm{JK}$, et al. (2008) Hyperbolic relationship between insulin secretion and sensitivity on oral glucose tolerance test. Obesity (Silver Spring) 16: 1901-1907.

24. Westwood M, Anderson LJ, Firmin DN, Gatehouse PD, Charrier CC, et al. (2003) A single breath-hold multiecho $\mathrm{T} 2 *$ cardiovascular magnetic resonance technique for diagnosis of myocardial iron overload. J Magn Reson Imaging 18: 33-39.

25. He T, Gatehouse PD, Smith GC, Mohiaddin RH, Pennell DJ, et al. (2008) Myocardial T2* measurements in ironoverloaded thalassemia: an in vivo study to investigate optimal methods of quantification. Magn Reson Med 60: 1082-1089.

26. Wood JC, Enriquez C, Ghugre N, Tyzka JM, Carson S, et al. (2005) MRI R2 and R2* mapping accurately estimates hepatic iron concentration in transfusion-dependent thalassemia and sickle cell disease patients. Blood 106: 14601465.

27. Borgna-Pignatti C, Rugolotto S, De Stefano P, Zhao H, Cappellini MD, et al. (2004) Survival and complications in patients with thalassemia major treated with transfusion and deferoxamine. Haematologica 89: 1187-1193.

28. Telfer PT, Prestcott E, Holden S, Walker M, Hoffbrand $\mathrm{AV}$, et al. (2000) Hepatic iron concentration combined with long-term monitoring of serum ferritin to predict complications of iron overload in thalassaemia major. $\mathrm{Br} J$ Haematol 110: 971-977.

29. Porter JB, Elalfy M, Taher A, Aydinok Y, Lee SH, et al. (2017) Limitations of serum ferritin to predict liver iron concentration responses to deferasirox therapy in patients with transfusion-dependent thalassaemia. Eur J Haematol 98: 280-288.

30. Bassett ML, Halliday JW, Powell LW (1986) Value of hepatic iron measurements in early haemochromatosis and determination of the critical iron level associated with fibrosis. Hepatology 6: 24-29.

31. Borgna-Pignatti C, Rugollotto S, DeStafano P, Piga A, Di Gregorio F, et al. (1998) Survival and disease complications in thalassemia major. Ann N Y Acad Sci 850: 227231.

32. Caprio S (2012) Development of type 2 diabetes mellitus in the obese adolescent: a growing challenge. Endocr Pract 18: 791-795.

33. Belhoul KM, Bakir ML, Saned MS, Kadhim AM, Musallam KM, et al. (2012) Serum ferritin levels and endocrinopathy in medically treated patients with $\beta$ thalassemia major. Ann Hematol 91: 1107-1114.

34. Au WY, Lam WW, Chu W, Tam S, Wong WK, et al. (2008) A T2* magnetic resonance imaging study of pancreatic iron over-load in thalassemia major. Haematologica 93: 116-119.

35. de Assis RA, Ribeiro AA, Kay FU, Rosemberg LA, Nomura $\mathrm{CH}$, et al. (2012) Pancreatic iron stores assessed by magnetic resonance imaging (MRI) in beta thalassemic patients. Eur J Radiol 81: 1465-1470.

36. Oudit GY, Trivieri MG, Khaper N, Liu PP, Backx PH (2006) Role of L-type Ca2+ channels in iron transport and iron-overload cardiomyopathy. J Mol Med (Berl) 84: 349364.

37. Noetzli LJ, Mittelman SD, Watanabe RM, Coates TD, Wood JC (2012) Pancreatic iron and glucose dysregulation in thalassemia major. Am J Hematol 87: 155-160.

38. Kahn SE (2003) The relative contributions of insulin resistance and beta-cell dysfunction to the pathophysiology of Type 2 diabetes. Diabetologia 46: 3-19.

39. Bergman RN, Ader M, Huecking K, Van Citters G (2002) Accurate assessment of beta-cell function: the hyperbolic correction. Diabetes 51 Suppl 1: S212-S220.

40. Bergman RN, Phillips LS, Cobelli C (1981) Physiologic evaluation of factors controlling glucose tolerance in man: measurement of insulin sensitivity and beta-cell glucose sensitivity from the response to intravenous glucose. $J$ Clin Invest 68: 1456-1467.

41. Kahn SE, Prigeon RL, McCulloch DK, Boyko EJ, Bergman RN, et al. (1993) Quantification of the relationship between insulin sensitivity and beta-cell function in human subjects. Evidence for a hyperbolic function. Diabetes 42: 1663-1672.

42. Retnakaran R, Qi Y, Goran MI, Hamilton JK (2009) Evaluation of proposed oral disposition index measures in relation to the actual disposition index. Diabet Med 26: 11981203. 